Published in final edited form as:

Alzheimer Dis Assoc Disord. 2020 ; 34(1): 18-24. doi:10.1097/WAD.0000000000000351.

\title{
Sleep Quality and Cognitive Function in Type 1 Diabetes: Findings from the Study of Longevity in Diabetes (SOLID)
}

\author{
Paola Gilsanz, ScD 1 , Mary E. Lacy, PhD ${ }^{1,2}$, Michal Schnaider Beeri, PhD ${ }^{3,4}$, Andrew J. \\ Karter, PhD ${ }^{1,5}$, Chloe Eng, MSPH ${ }^{1,2}$, Rachel A. Whitmer, PhD ${ }^{6,1,2}$ \\ ${ }^{1}$ Kaiser Permanente Northern California Division of Research, CA \\ ${ }^{2}$ Department of Epidemiology and Biostatistics, University of California San Francisco, CA \\ ${ }^{3}$ Department of Psychiatry, Icahn School of Medicine at Mount Sinai, NY \\ ${ }^{4}$ The Joseph Sagol Neuroscience Center, Sheba Medical Center, Israel \\ ${ }^{5}$ Department of Health Services, University of Washington, Seattle, WA \\ ${ }^{6}$ Department of Public Health Science, University of California Davis School of Medicine, CA
}

\section{Abstract}

Study Objectives: To examine the association between sleep quality and global and domainspecific cognitive function among older individuals with type 1 diabetes (T1D).

\begin{abstract}
Methods: We evaluated 695 individuals with T1D ages $\ 60$ that participated in the baseline assessment of the Study of Longevity in Diabetes (SOLID), which captured subjective sleep quality (Pittsburg Sleep Quality Index) and global and domain-specific (language, executive function, episodic memory, and simple attention) cognitive function. Multivariable linear regressions estimated the associations between sleep quality quartiles and overall and domainspecific cognitive function adjusting for age, sex, race/ethnicity, education, depressive symptoms, and severe hypoglycemic episodes. Sensitivity analyses examined the associations between aspects of sleep quality and global cognitive function.
\end{abstract}

Results: The worst sleep quality quartile was associated with lower global cognition ( $\beta=-0.08$; $95 \%$ CI: $-0.17,-0.01$ ) and lower executive function ( $\beta=-0.17,95 \%$ CI: $-0.30,-0.03$ ) compared to the best quartile of sleep quality adjusting for demographics and comorbidities. Sleep quality was not associated with language, episodic memory, or simple attention. Sleep medications and daytime dysfunction were most strongly associated with global cognition.

Conclusions: Our results suggest that sleep quality may be a modifiable risk factor for global cognitive function and executive function among elderly individuals with T1D.

\section{Keywords}

type 1 diabetes; sleep quality; cognitive function

Corresponding author: Paola Gilsanz, ScD, Kaiser Permanente Division of Research, 2000 Broadway, Oakland, CA 94612 , Paola.Gilsanz@kp.org, Phone: (510) 891-3482.

Conflicts of interest: All authors report no conflicts of interest. 
An estimated 50 to 70 million Americans are afflicted by sleep-related problems ${ }^{1}$ and approximately $35.2 \%$ of US adults report short sleep duration (i.e. $<7$ hours in past 24 hours $)^{2}$. The far-reaching negative impacts of sleep disorders and short sleep duration are exemplified by their associations with elevated risk of several health outcomes, including obesity $^{3}$, hypertension ${ }^{3}$, stroke ${ }^{3,4}$, and all-cause mortality ${ }^{3,4}$. Sleep disorders, short sleep duration, and sleep quality have also been associated with lower levels of cognitive function in general populations ${ }^{5-10}$ However, it remains unclear whether specific cognitive domains are impacted differentially by sleep quality ${ }^{9-11}$.

Poor quality sleep burdens $31-41 \%$ of adults with type 1 diabetes (T1D) $)^{12-14}$, as compared to $39-60 \%$ of adults with type 2 diabetes (T2D) ${ }^{14-16}$, and $6.5-38 \%$ of adults without diabetes $^{12,17,18}$. Poor sleep quality is more severe among adults with T1D compared to the general population. ${ }^{19}$ Furthermore, T1D has been consistently associated with cognitive impairment. ${ }^{20,21}$ However, the majority of this work has focused on individuals with T1D who are middle aged or younger. ${ }^{20}$ As the life expectancy of individuals with T1D increases, a better understanding of cognitive function among older adults with T1D is needed. Identifying which risk factors for cognitive decline among individuals with T1D are modifiable is especially important for this population given the considerable burden and complexity of self-care management. It is unknown if sleep quality is associated with cognitive impairment among older adults with T1D.

In this study we examine the association between sleep quality and global and domainspecific cognitive function in a sample of individuals with T1D ages 60 and older. Cognitive data were collected by a thorough neuropsychological battery selected to assess global cognitive function as well as a range of cognitive domains including language, executive function, episodic memory, and simple attention. In sensitivity analyses we examine the association between various aspects of sleep quality and overall and domain specific cognitive function.

\section{Methods}

\section{Study population.}

These analyses included members of Kaiser Permanente Northern California (KPNC) with T1D who participated in the Study of Longevity in Diabetes (SOLID). SOLID is a prospective cohort study of diabetes and aging that recruited KPNC members ages $ð 00$. Potential participants were identified in electronic medical records using International Classification of Diseases (ICD)-9 and ICD-10 codes for T1D (250.x1, 250.x3, or E10.x) and T2D (250.x0, 250.x2, E11.x). Members were classified as having T1D if at least 75\% of diagnostic codes related to diabetes were for T1D and they were prescribed insulin. Medical providers of potential participants were contacted and could provide information regarding whether a member met inclusion criteria or refuse their patient's participation for any reason. Potential participants for whom a medical provider did not report a refusal or ineligibility were sent an introductory letter. Potential participants that did not refuse by mail were contacted for a phone screening during which they were asked questions regarding type of diabetes diagnosis (T1D or T2D), age of diagnosis, and timing of insulin use initiation. Individuals who reported a diagnosis of T1D at ages $\$ 30$ were classified as having T1D. 
Manual medical record review was coducted for participants reporting the onset of T1D at ages $\geq 31$ years to confirm T1D status. 805 individuals with T1D were enrolled in SOLID and completed baseline interviews.

This study was approved by the KPNC Internal Review Board and informed consent was provided by all enrolled participants.

\section{Self-reported sleep quality.}

Self-reported sleep quality in the past month was captured at the baseline interview by a modified version of the Pittsburgh Sleep Quality Index (PSQI) ${ }^{22}$ that excluded an item assessing snoring frequency. The PSQI covers 7 components of sleep quality each of which has scores ranging from 0 (no difficulty) to 3 (severe difficulty): a one-item measure of subjective sleep quality, sleep disturbances, sleep medication use, daytime dysfunction, sleep latency, sleep duration, and sleep efficiency. The subjective sleep quality component was assessed through the following question: "During the past month, how would you rate your sleep quality overall?" and included the following response options: very good, fairly good, fairly bad, and very bad. Items assessing the frequency of sleep disturbances (e.g. too hot or cold, nightmares, pain), sleep medication use, and daytime dysfunction included the following response options: not during the past month, less than once a week, once or twice a week, three or more times a week. Sleep latency was captured through items assessing the average length of time spent transitioning from wakefulness to sleep and how often participants couldn't fall asleep within 30 minutes (response options: not during the past month, less than once a week, once or twice a week, three or more times a week). Average sleep duration during the past month was captured continuously in hours and operationalized as a continuous variable and as binary variables using the following thresholds: $\mathbf{5}$ hours, $\mathbf{6}$ hours, $\checkmark 7$ hours, and $\geq 9$ hours of sleep. Sleep efficiency was defined as the percent of time in bed an individual was asleep. Global PSQI scores range from 0 to 21 with higher scores indicating worse sleep quality. Compared to polysomnographic results, a score of greater than 5 denotes poor sleep with a sensitivity and specificity of $90 \%$ and $87 \% .^{22}$ Approximately $68 \%$ of the sample had poor sleep quality using a threshold of 5. Global PSQI was divided into quartiles, with higher quartiles reflecting worst sleep quality, to allow for a possible non-linear relationship between sleep quality and cognitive function.

\section{Cognitive Function.}

A comprehensive cognitive battery was administrated to all participants and, similar to a previous study among individuals with $\mathrm{T} 2 \mathrm{D}$, a factor analysis revealed four cognitive domains. ${ }^{23}$ The language factor encompassed phonemic fluency (F and L), category fluency (animals and vegetables), list sorting (two alternative lists), and Multilingual Naming Test (MINT). The executive function domain encompassed the Trail Making Test (A and B), Digit Symbol Substitution, and the Stroop Color and Word Tests. The episodic memory domain encompassed the Word List Learning Test (immediate and delayed) and the Benson Complex Figure Copy (immediate and delayed). The simple attention domain encompassed the Diamond and TMX cancellation tests. 
Overall and domain-specific scores were estimated using standardized (mean $=0$; standard deviations $(\mathrm{SD})=1$ ) cognitive function test scores. Domain-specific scores were estimated for individuals who completed at least $50 \%$ of the relevant tests. Global cognitive function scores were estimated as the average of the four domain specific scores for individuals who completed at least $50 \%$ of all cognitive function tests. Impaired global or domain-specific scores were defined as scores less than 1.5 standard deviation below the mean.

\section{Covariates.}

Date of birth and interview date were used to estimate age at interview. Self-reported race/ ethnicity was obtained during baseline interviews and re-categorized into the following group: White, Black, Hispanic, Asian, other racial/ethnic group, and refuse/don't know. Sex was obtained from KPNC records. The frequency of severe hypoglycemic episodes during the past year was captured at baseline using the following item: "In the past year, how often have you had severe hypoglycemic episodes? (Episodes where you were unconscious or had a seizure and needed glucagon or intravenous glucose)". The frequency of severe hypoglycemic episodes was recoded into 0,1 or 2 , and $\geq 3$. The Beck Depression Inventory was implemented to capture depressive symptoms. The scale is comprised of 21 items, each of which elicits a rating of symptom severity on a scale of 0 to 3 , that are summed to provide a total score ranging from 0 to $63 .{ }^{24}$ Individuals with a total score of at least 13 were considered to have elevated depressive symptoms based on prior work demonstrating a moderate to high sensitivity $(0.85)$ and specificity $(0.88)$ of this threshold compared to a diagnosis of current major depression according to the Diagnostic and Statistical Manual of Mental Disorders (DSM)-III-R. ${ }^{25}$ Missing indicators were used for people missing values on depressive symptoms $(\mathrm{n}=18)$ and individuals missing data on race/ethnicity, education, or severe hypoglycemic episodes were excluded (see below).

\section{Analytic sample.}

These analyses exclude 67 people missing information on sleep measure components, 35 people who completed less than $50 \%$ of all cognitive testing, 4 people with missing or unknown race/ethnicity, 2 people missing information regarding educational attainment, and 2 people missing information regarding severe hypoglycemic episodes. The final sample for analyses examining associations with global cognitive function encompassed 695 individuals. The following number of people completed at least $50 \%$ of domain-specific cognitive tests for the relevant domain and are included in analyses specific to those domains: language: 680 participants; executive function: 680 participants; episodic memory: 661 participants; simple attention: 679 participants.

\section{Analyses.}

The distribution of demographics, elevated depressive symptoms, and severe hypoglycemic episodes was examined overall and by sleep quality quartile. The distribution of sleep quality characteristics was examined overall and by sex. In preliminary analyses, Pearson correlations examined the unadjusted association between overall sleep quality and specific sleep quality aspects with overall cognitive function. 
Multivariable linear regression estimated the association between sex and cognition (overall and domain specific). Multivariable linear regressions estimated the associations between PSQI score quartiles (reference=best sleep quality quartile) and performance on overall and domain-specific cognitive function. Confounders were sequentially added to models in two groups: 1) demographics (age, sex, race/ethnicity, and education); and 2) comorbidities (elevated depressive symptoms, severe hypoglycemic episodes). Sensitivity analyses estimated the odds of global and domain-specific impaired cognitive function (defined as 1.5 SD below mean) associated with sleep quality quartiles.

Linear and logistic multivariable regression examined the associations between each of the 7 aspects of sleep quality and performance on global cognitive function (continuous) and impaired global cognition (binary) adjusting for demographics and comorbidities. Sleep duration was operationalized continuously and with thresholds at 5 hours, 56 hours, 57 hours, $\geq 9$ hours.

\section{Results}

The mean PSQI score was $8.16(\mathrm{SD}=2.8$ ) and PSQI quartiles had the following range of scores: $1^{\text {st }}$ quartile (best sleep quality quartile) included scores $0-6,2^{\text {nd }}$ quartile included a score of $7,3^{\text {rd }}$ quartile included scores of 8 or 9 , and the $4^{\text {th }}$ quartile (worst sleep quality quartile) included scores of $\geq 10$. The mean age at baseline interview was 67.3 years ( $\mathrm{SD}=6.4$ years) and participants were predominantly White (86.3\%) and had at least a college degree (62.6\%) (Table 1). Elevated depressive symptoms were reported by $7.5 \%$ of the participants and most participants reported no severe hypoglycemic episodes during the prior year. A total of $7.2 \%$ of participant had impaired global cognition and the prevalence of impairment within specific domains ranged from $6.3 \%$ to $7.9 \%$ (6.3\% impaired language, $7.1 \%$ impaired attention, $7.9 \%$ impaired executive function, $7.4 \%$ impaired episodic memory). Women had higher scores for PSQI (t-test p-value $<0.0001$ ), sleep latency (t-test p-value $<0.0001$ ), sleep efficiency ( $\mathrm{p}$-value $=0.03$ ), sleep disturbances ( $\mathrm{t}$-test $\mathrm{p}$-value $<0.0001$ ), and sleep medication (t-test $\mathrm{p}$-value $<0.01$ ) than men (Table 2). There was no difference across sexes in sleep hours operationalized continuously (t-test p-value $=0.73$ ) or as $\$ \mathbf{s}$ hours, 6 hours, 7 hours, 8 hours, or $\geq 9$ hours (chi-square $\mathrm{p}$-value $=0.56$ ) or in daytime dysfunction (t-test $\mathrm{p}$-value $=0.14$ ). Sleep quality and its components were weakly correlated with global cognitive function ( $r$ range: 0.06 to -0.09 ; Supplemental Table 1 ).

Compared to men, women had a higher global cognitive score ( $\beta=0.22,95 \%$ CI: $0.15,0.29)$, executive function ( $\beta=0.13,95 \%$ CI: $0.02,0.24)$, episodic memory ( $\beta=0.35,95 \%$ CI: 0.25 , $0.44)$, and simple attention $(\beta=0.29,95 \%$ CI: $0.17,0.41)$ adjusting for demographics. There was no difference by sex in scores in the language domain ( $\beta=0.08,95 \% \mathrm{CI}:-0.02,0.18$ ).

Compared to individuals in the best sleep quality quartile, individuals in the worst quartile of sleep quality scored 0.08 units lower on the global cognition score $(\beta=-0.08 ; 95 \%$ Confidence Interval (CI): $-0.17,-0.01$; Table 3 ) in models adjusting for demographics, elevated depressive symptoms, and severe hypoglycemic episodes. There was no association between other quartiles of sleep quality and global cognitive function. In domain-specific analyses, individuals in the worst sleep quality quartile had -0.17 units lower scores of 
executive function ( $\beta=-0.17 ; 95 \% \mathrm{CI}:-0.30,-0.03$ ) compared to individuals in the best sleep quality quartile. Although the associations between sleep quality and the other cognitive domains were in the same direction as that of the sleep quality-executive function relationship, the differences between the worst and best quartile of sleep quality were not significant (language ( $\beta=-0.07 ; 95 \% \mathrm{CI}:-0.20,0.06)$, episodic memory $(\beta=-0.11 ; 95 \% \mathrm{CI}$ $-0.23,0.02)$, simple attention $(\beta=-0.004 ; 95 \% \mathrm{CI}-0.16,0.17))$.

While the odds of impaired global cognitive function (i.e. scoring below 1.5 standard deviations below the mean) was elevated for the worst sleep quality quartile compared to the best sleep quality quartile, this difference was not significant (odds ratio $(\mathrm{OR})=1.79 ; 95 \%$ CI: $0.82,3.91)$. The worst quartile of sleep quality had more than double the odds (OR=2.49; 95\% CI: 1.04, 5.98; Table 4) of impaired language compared to the best sleep quality quartile adjusting for demographics, elevated depressive symptoms, and severe hypoglycemic episodes. The worst sleep quality quartile was associated with elevated odds of impairment in the other domains but these differences were not significant (impaired executive function ( $\mathrm{OR}=1.83 ; 95 \%$ CI: $0.86,3.91)$, impaired episodic memory ( $\mathrm{OR}=1.42$; 95\% CI: 0.66, 3.03), impaired simple attention (OR=1.96; 95\% CI: 0.85, 4.50)).

In models examining the association between specific aspects of sleep quality and global cognitive function (Table 5), adjusting for demographics, elevated depressive symptoms, and severe hypoglycemic episodes, sleep medication use $(\beta=-0.03 ; 95 \%$ CI: $-0.06,-0.002)$ and daytime dysfunction ( $\beta=-0.07 ; 95 \% \mathrm{CI}:-0.12,-0.02$ ) were associated with lower global cognition. There was no evidence of an association between subjective sleep quality as reported in one item ( $\beta=0.02 ; 95 \% \mathrm{CI}:-0.02,0.07)$, sleep latency $(\beta=-0.01 ; 95 \% \mathrm{CI}$ : $-0.05,0.03)$, sleep efficiency ( $\beta=-0.01 ; 95 \%$ CI: $-0.04,0.03$ ), or sleep disturbances ( $\beta=$ -0.03 ; 95\% CI: $-0.08,0.02)$ and global cognition. There was no evidence that sleep duration, as a continuous measure $(\beta=-0.01 ; 95 \% \mathrm{CI}:-0.04,0.01)$ or using thresholds of sleep of $\leq 5$ hours $(\beta=0.06$; 95\% CI: $-0.04,0.17)$, $\$ 6$ hours $(\beta=0.0002 ; 95 \%$ CI: $-0.07,0.07$ ), $\leq 7$ hours ( $\beta=0.06$; 95\% CI: $-0.01,0.13)$, or $\geq 9$ hours $(\beta=0.04$; 95\% CI: $-0.07,0.15)$, was associated with global cognition. None of the specific aspects of sleep quality were associated with impaired global cognition.

\section{Conclusions}

Similar to prior work demonstrating an association between sleep quality and cognitive function in the general population and among people with T2D, this first study of sleep quality and cognition in elderly individuals with T1D detected a threshold effect in which poor sleep quality was associated with lower global cognition. Individuals in the worst quartile of sleep quality had lower global cognition compared to their counterparts in the best quartile of sleep quality. This relationship seemed to be primarily driven by the association between poor sleep quality and executive function. Sleep quality was not associated with performance on cognitive tests related to language, episodic memory, or simple attention. Individuals in the worst quartile of sleep quality had over double the odds of impaired language adjusting for age, sex, race/ethnicity, education, elevated depressive symptoms, and severe hypoglycemic episodes. Sleep medication use and daytime dysfunction were the aspects of poor-quality sleep associated lower global cognitive 
function. Our findings are consistent with a study among 162 adults with prediabetes or T2D that showed an association between higher sleep efficiency and higher global cognition. ${ }^{26}$ Each one-point increase in sleep efficiency among people with prediabetes or T2D was associated with a 0.09 point increase in the Montreal Cognitive Assessment (MoCA) score. Consistent with our findings, the study did not provide evidence of an association between sleep duration, measured continuously, and global cognitive function. Our findings are also consistent with prior prospective studies ${ }^{5,9}$ and cross-sectional studies ${ }^{6,10}$ in the general population that have demonstrated an association between sleep quality, captured by the $\mathrm{PSQI}^{5,9,10}$ or other composite variables ${ }^{6}$, and global cognitive function. However, the largest prospective study examining a composite of sleep quality and cognitive function in the general population included 2,822 men 65 years old and older and did not see an association between PSQI and global cognitive function measured by the Modified Mini-Mental State Examination (3MS) after an average of 3.4 years of follow-up. ${ }^{11}$ Another large prospective study of 1,664 men and women ages 65 and older found an association between PSQI and global cognition measured one year later in men but not women. ${ }^{9}$ Among men, each additional point on the PSQI was associated with $17 \%$ greater odds of incident global cognitive impairment. Possible reasons for inconsistencies in findings across studies in the general population include differences in follow-up time and measures of cognitive function.

It is unclear how overall measures of sleep quality are associated with specific domains of cognitive function as few studies have examined these relationships. ${ }^{9-11}$ Consistent with our results, a study of 157 elderly men and women and a study of 2,822 elderly men have both demonstrated a relationship between poor sleep quality and lower scores on the Trails Making Test-Part $\mathrm{B}^{10,11}$, which is a component of the executive function domain in our study. In the sample of 157 elderly individuals, there were also associations between poor sleep quality and lower scores on tests related to working memory and abstract problem solving but no association with tests related to episodic memory, inhibitory function, or abstract problem solving. ${ }^{10}$ Different choices of cognitive tests make it difficult to compare results across studies and may explain some of the inconsistencies in findings.

Much of the prior research to date has examined the association between specific aspects of sleep quality (as opposed to an overall measure of sleep quality such as the PSQI) and cognitive function. Sleep disturbances and long sleep duration have been associated with global cognitive impairment in women while short sleep duration and sleep efficiency were associated with global cognitive impairment in men. ${ }^{9}$ In line with our findings, daytime sleepiness has been associated with worse cognitive function in some studies ${ }^{7,27,28}$ although other studies have not shown an association ${ }^{9,11}$. Inconsistent with our findings, sleep efficiency has been repeatedly associated with global cognition ${ }^{5,9,26,29}$; though in one study this association was found only among men and not women ${ }^{9}$. Consistent with some prior studies $5,9,27,30$, we found no association between sleep latency and global cognitive function. Our results are also consistent with prior studies demonstrating no association between a continuous measures of sleep duration and global cognitive function. ${ }^{1,26,29}$ However, our study is inconsistent with prior work showing an association with short sleep duration (i.e. 5 hours ${ }^{5,9}, 56$ hours $^{6}, 56.5$ hours $^{27}$, or $\leq 7$ hours $^{8}$ ) and cognitive function. Our study is consistent with prior work demonstrating no association between long sleep duration (i.e $\geq 9$ hours ${ }^{5,27}$ ) and cognition, though other studies have shown an association in a sample of both 
sex ${ }^{6}$, women but not men ${ }^{9}$, or with dementia ${ }^{31}$. Differences in study type, sample populations, sleep quality measures, and cognitive function assessments hinder direct comparisons across studies.

Strengths of this study include a large sample of elderly with T1D, the ability to examine specific aspects of poor-quality sleep as well as overall sleep quality, and the ability to examine domains of cognitive function. Limitations of the study include reliance on selfreported measures of sleep quality as opposed to objective measures captured by polysomnography or actigraphy. Additionally, there is no neuroimaging or pathology data to examine the neuropathological substrates linking subjective sleep quality with cognition. Furthermore, at this time, our analyses include only baseline data from the SOLID study, which is ongoing, and therefore the directionality of the association between sleep quality and cognition cannot be ensured. The prevalence of impaired global cognition was $7 \%$ in this sample and, although the direction of the association between sleep quality and cognitive impairment was similar to analyses examining cognition as a continuous variable, analyses examining cognitive impairment may be underpowered. Examining the longitudinal relationship between sleep quality and cognitive function will be the focus of future research as more data is collected. Finally, our study is collecting data on participants with T2D and participants without diabetes so direct comparisons of associations of sleep quality with cognition to participants without T1D will be available in the future.

Although the exact pathway through which sleep quality is associated with cognitive function remains unknown, several mechanisms have been hypothesized to be at play in the general population. Some studies suggest that sleep is involved in amyloid beta (A $\beta$ ) clearance $^{32,33}$ and excessive daytime sleepiness has been associated with increased longitudinal $A \beta$ accumulation ${ }^{34,35}$. Poor sleep quality has also been associated with higher levels of pro-inflammatory cytokines ${ }^{36}$ and cortisol ${ }^{37}$, both of which have been associated with cognitive function or decline ${ }^{38,39}$. Studies have also linked sleep quality to white matter microstructure ${ }^{40}$ and the presence and severity of white matter hyperintensities ${ }^{41}$, which are also associated with cognitive function. Among individuals with T1D, poor quality sleep has been associated with higher hemoglobin $\mathrm{A} 1 \mathrm{c}(\mathrm{HbA} 1 \mathrm{c})$ levels ${ }^{19}$, and $\mathrm{HbA} 1 \mathrm{c}$ has been associated with cognition ${ }^{42}$.

To our knowledge this is the first study to examine the association of sleep quality and cognitive function among older adults with T1D. Healthy aging for individuals with T1D requires vigilance and extreme attention to self-care. Further research should examine sleep quality as a modifiable risk factor for cognitive function that, if improved, may increase individuals' ability to manage T1D in late-life.

\section{Supplementary Material}

Refer to Web version on PubMed Central for supplementary material.

\section{Acknowledgments}

Funding: The authors gratefully acknowledge funding from the National Institutes on Aging (NIA R01 AG047500; Dr. Whitmer). Dr. Lacy and Ms. Eng were supported by the UCSF Training for Research on Aging and Chronic 
Disease (T32 AG049663). Dr. Lacy was also supported through contract PPRN-1306-04709 from the PatientCentered Outcomes Research Institute (PCORI), PPRN-1306-04709.

$\begin{array}{ll}\text { Abbreviations } & \\ \text { 3MS } & \text { Modified Mini-Mental State Examination } \\ \text { A } \beta & \text { Amyloid beta } \\ \text { CI } & \text { Confidence Interval } \\ \text { DSM } & \text { Diagnostic and Statistical Manual of mental Disorders } \\ \text { HbA1c } & \text { hemoglobin A1c } \\ \text { ICD } & \text { International Classification of Diseases } \\ \text { PSQI } & \text { Pittsburgh Sleep Quality Index } \\ \text { MINT } & \text { Multilingual Naming Test } \\ \text { MoCA } & \text { Montreal Cognitive Assessment } \\ \text { OR } & \text { Odds ratio } \\ \text { SD } & \text { Standard deviations } \\ \text { SOLID } & \text { Study of Longevity in Diabetes } \\ \text { T1D } & \text { Type 1 diabetes } \\ \text { T2D } & \text { Type 2 diabetes } \\ & \end{array}$

\section{References}

1. National Center on Sleep Disorders R, Trans NIHSRCC. 2003 national sleep disorders research plan [Bethesda Md.]: U.S. Dept. of Health and Human Services, National Institutes of Health, National Heart, Lung, and Blood Institute, National Center on Sleep Disorders Research, Trans-NIH Sleep Research Coordinating Committee; 2003.

2. National Center for Chronic Disease Prevention and Health Promotion DoPH. Short Sleep Duration Among US Adults. Data and Statistics 2017; https://www.cdc.gov/sleep/data_statistics.html. Accessed 11/28/2018, 2018.

3. Itani O, Jike M, Watanabe N, Kaneita Y. Short sleep duration and health outcomes: a systematic review, meta-analysis, and meta-regression. Sleep medicine. 2017;32:246-256. [PubMed: 27743803]

4. Yin J, Jin X, Shan Z, et al. Relationship of Sleep Duration With All-Cause Mortality and Cardiovascular Events: A Systematic Review and Dose-Response Meta-Analysis of Prospective Cohort Studies. Journal of the American Heart Association. 2017;6(9) e005947. [PubMed: 28889101]

5. Niu J, Han H, Wang Y, Wang L, Gao X, Liao S. Sleep quality and cognitive decline in a community of older adults in Daqing City, China. Sleep medicine. 2016;17:69-74. [PubMed: 26847977]

6. Gildner TE, Liebert MA, Kowal P, Chatterji S, Snodgrass JJ. Associations between sleep duration, sleep quality, and cognitive test performance among older adults from six middle income countries: results from the Study on Global Ageing and Adult Health (SAGE). Journal of clinical sleep medicine : JCSM : official publication of the American Academy of Sleep Medicine.

2014;10(6):613-621. [PubMed: 24932140] 
7. Foley D, Monjan A, Masaki K, et al. Daytime Sleepiness Is Associated with 3-Year Incident Dementia and Cognitive Decline in Older Japanese-American Men. Journal of the American Geriatrics Society. 2001;49(12):1628-1632. [PubMed: 11843995]

8. Virta JJ, Heikkila K, Perola M, et al. Midlife sleep characteristics associated with late life cognitive function. Sleep. 2013;36(10):1533-1541, 1541A. [PubMed: 24082313]

9. Potvin O, Lorrain D, Forget H, et al. Sleep quality and 1-year incident cognitive impairment in community-dwelling older adults. Sleep. 2012;35(4):491-499. [PubMed: 22467987]

10. Nebes RD, Buysse DJ, Halligan EM, Houck PR, Monk TH. Self-reported sleep quality predicts poor cognitive performance in healthy older adults. J Gerontol B Psychol Sci Soc Sci. 2009;64(2):180-187. [PubMed: 19204069]

11. Blackwell T, Yaffe K, Laffan A, et al. Associations of objectively and subjectively measured sleep quality with subsequent cognitive decline in older community-dwelling men: the MrOS sleep study. Sleep. 2014;37(4):655-663. [PubMed: 24899757]

12. van Dijk M, Donga E, van Dijk JG, et al. Disturbed subjective sleep characteristics in adult patients with long-standing type 1 diabetes mellitus. Diabetologia. 2011;54(8):1967-1976. [PubMed: 21573904]

13. Denic-Roberts H, Costacou T, Orchard TJ. Subjective sleep disturbances and glycemic control in adults with long-standing type 1 diabetes: The Pittsburgh's Epidemiology of Diabetes Complications study. Diabetes Res Clin Pract. 2016;119:1-12. [PubMed: 27415404]

14. Nefs G, Donga E, van Someren E, Bot M, Speight J, Pouwer F. Subjective sleep impairment in adults with type 1 or type 2 diabetes: Results from Diabetes MILES--The Netherlands. Diabetes Res Clin Pract. 2015;109(3):466-475. [PubMed: 26264411]

15. Aribas A, Kayrak M, Tekinalp M, et al. The relationship between serum asymmetric dimethylarginine levels and subjective sleep quality in normotensive patients with type 2 diabetes mellitus. Korean J Intern Med. 2015;30(3):316-324. [PubMed: 25995662]

16. Reutrakul S, Hood MM, Crowley SJ, et al. Chronotype is independently associated with glycemic control in type 2 diabetes. Diabetes Care. 2013;36(9):2523-2529. [PubMed: 23637357]

17. Chen X, Wang R, Zee P, et al. Racial/Ethnic Differences in Sleep Disturbances: The Multi-Ethnic Study of Atherosclerosis (MESA). Sleep. 2015;38(6):877-888. [PubMed: 25409106]

18. Lacruz ME, Schmidt-Pokrzywniak A, Dragano N, et al. Depressive symptoms, life satisfaction and prevalence of sleep disturbances in the general population of Germany: results from the Heinz Nixdorf Recall study. BMJ Open. 2016;6(1):e007919.

19. Reutrakul S, Thakkinstian A, Anothaisintawee T, et al. Sleep characteristics in type 1 diabetes and associations with glycemic control: systematic review and meta-analysis. Sleep medicine. 2016;23:26-45. [PubMed: 27692274]

20. Brands AM, Biessels GJ, de Haan EH, Kappelle LJ, Kessels RP. The effects of type 1 diabetes on cognitive performance: a meta-analysis. Diabetes Care. 2005;28(3):726-735. [PubMed: 15735218]

21. Li W, Huang E, Gao S. Type 1 Diabetes Mellitus and Cognitive Impairments: A Systematic Review. J Alzheimers Dis. 2017;57(1):29-36. [PubMed: 28222533]

22. Buysse DJ, Reynolds CF 3rd, Monk TH, Berman SR, Kupfer DJ. The Pittsburgh Sleep Quality Index: a new instrument for psychiatric practice and research. Psychiatry research. 1989;28(2):193-213. [PubMed: 2748771]

23. Beeri MS, Ravona-Springer R, Moshier E, et al. The Israel Diabetes and Cognitive Decline (IDCD) study: Design and baseline characteristics. Alzheimer's \& dementia : the journal of the Alzheimer's Association. 2014;10(6):769-778.

24. Beck AT, Ward CH, Mendelson M, Mock J, Erbaugh J. An inventory for measuring depression. Arch Gen Psychiatry. 1961;4(6):561-571. [PubMed: 13688369]

25. Lustman PJ, Clouse RE, Griffith LS, Carney RM, Freedland KE. Screening for depression in diabetes using the Beck Depression Inventory. Psychosomatic medicine. 1997;59(1):24-31. [PubMed: 9021863]

26. Saetung S, Nimitphong H, Siwasaranond N, et al. The relationship between sleep and cognitive function in patients with prediabetes and type 2 diabetes. Acta Diabetol. 2018;55(9):917-925. [PubMed: 29872969] 
27. Keage HA, Banks S, Yang KL, Morgan K, Brayne C, Matthews FE. What sleep characteristics predict cognitive decline in the elderly? Sleep medicine. 2012;13(7):886-892. [PubMed: 22560827]

28. Gabelle A, Gutierrez LA, Jaussent I, et al. Excessive Sleepiness and Longer Nighttime in Bed Increase the Risk of Cognitive Decline in Frail Elderly Subjects: The MAPT-Sleep Study. Frontiers in aging neuroscience. 2017;9:312. [PubMed: 29033827]

29. Blackwell T, Yaffe K, Ancoli-Israel S, et al. Poor Sleep Is Associated With Impaired Cognitive Function in Older Women: The Study of Osteoporotic Fractures. The Journals of Gerontology Series A: Biological Sciences and Medical Sciences. 2006;61(4):405-410.

30. Haba-Rubio J, Marti-Soler H, Tobback N, et al. Sleep characteristics and cognitive impairment in the general population: The HypnoLaus study. Neurology. 2017;88(5):463-469. [PubMed: 28039311]

31. Benito-Leon J, Bermejo-Pareja F, Vega S, Louis ED. Total daily sleep duration and the risk of dementia: a prospective population-based study. European journal of neurology. 2009;16(9):990997. [PubMed: 19473367]

32. Kang JE, Lim MM, Bateman RJ, et al. Amyloid-beta dynamics are regulated by orexin and the sleep-wake cycle. Science (New York, NY). 2009;326(5955):1005-1007.

33. Ooms S, Overeem S, Besse K, Rikkert MO, Verbeek M, Claassen JA. Effect of 1 night of total sleep deprivation on cerebrospinal fluid beta-amyloid 42 in healthy middle-aged men: a randomized clinical trial. JAMA Neurol. 2014;71(8):971-977. [PubMed: 24887018]

34. Carvalho DZ, St Louis EK, Knopman DS, et al. Association of Excessive Daytime Sleepiness With Longitudinal beta-Amyloid Accumulation in Elderly Persons Without Dementia. JAMA Neurol. 2018;75(6):672-680. [PubMed: 29532057]

35. Spira AP, An Y, Wu MN, et al. Excessive daytime sleepiness and napping in cognitively normal adults: associations with subsequent amyloid deposition measured by PiB PET. Sleep. 2018;41(10).

36. Milrad SF, Hall DL, Jutagir DR, et al. Poor sleep quality is associated with greater circulating proinflammatory cytokines and severity and frequency of chronic fatigue syndrome/myalgic encephalomyelitis (CFS/ME) symptoms in women. Journal of neuroimmunology. 2017;303:4350. [PubMed: 28038892]

37. Castro-Diehl C, Diez Roux AV, Redline S, Seeman T, Shrager SE, Shea S. Association of Sleep Duration and Quality With Alterations in the Hypothalamic-Pituitary Adrenocortical Axis: The Multi-Ethnic Study of Atherosclerosis (MESA). J Clin Endocrinol Metab. 2015;100(8):3149_ 3158. [PubMed: 26046965]

38. Schram MT, Euser SM, de Craen AJ, et al. Systemic markers of inflammation and cognitive decline in old age. J Am Geriatr Soc. 2007;55(5):708-716. [PubMed: 17493190]

39. Comijs HC, Gerritsen L, Penninx BW, Bremmer MA, Deeg DJ, Geerlings MI. The association between serum cortisol and cognitive decline in older persons. The American journal of geriatric psychiatry : official journal of the American Association for Geriatric Psychiatry. 2010;18(1):4250. [PubMed: 20094017]

40. Sexton CE, Zsoldos E, Filippini N, et al. Associations between self-reported sleep quality and white matter in community-dwelling older adults: A prospective cohort study. Human brain mapping. 2017;38(11):5465-5473. [PubMed: 28745016]

41. Del Brutto OH, Mera RM, Zambrano M, Lama J, Del Brutto VJ, Castillo PR. Poor sleep quality and silent markers of cerebral small vessel disease: a population-based study in communitydwelling older adults (The Atahualpa Project). Sleep medicine. 2015;16(3):428-431. [PubMed: 25681211]

42. Luchsinger JA, Palmas W, Teresi JA, et al. Improved diabetes control in the elderly delays global cognitive decline. The journal of nutrition, health \& aging. 2011;15(6):445-449. 


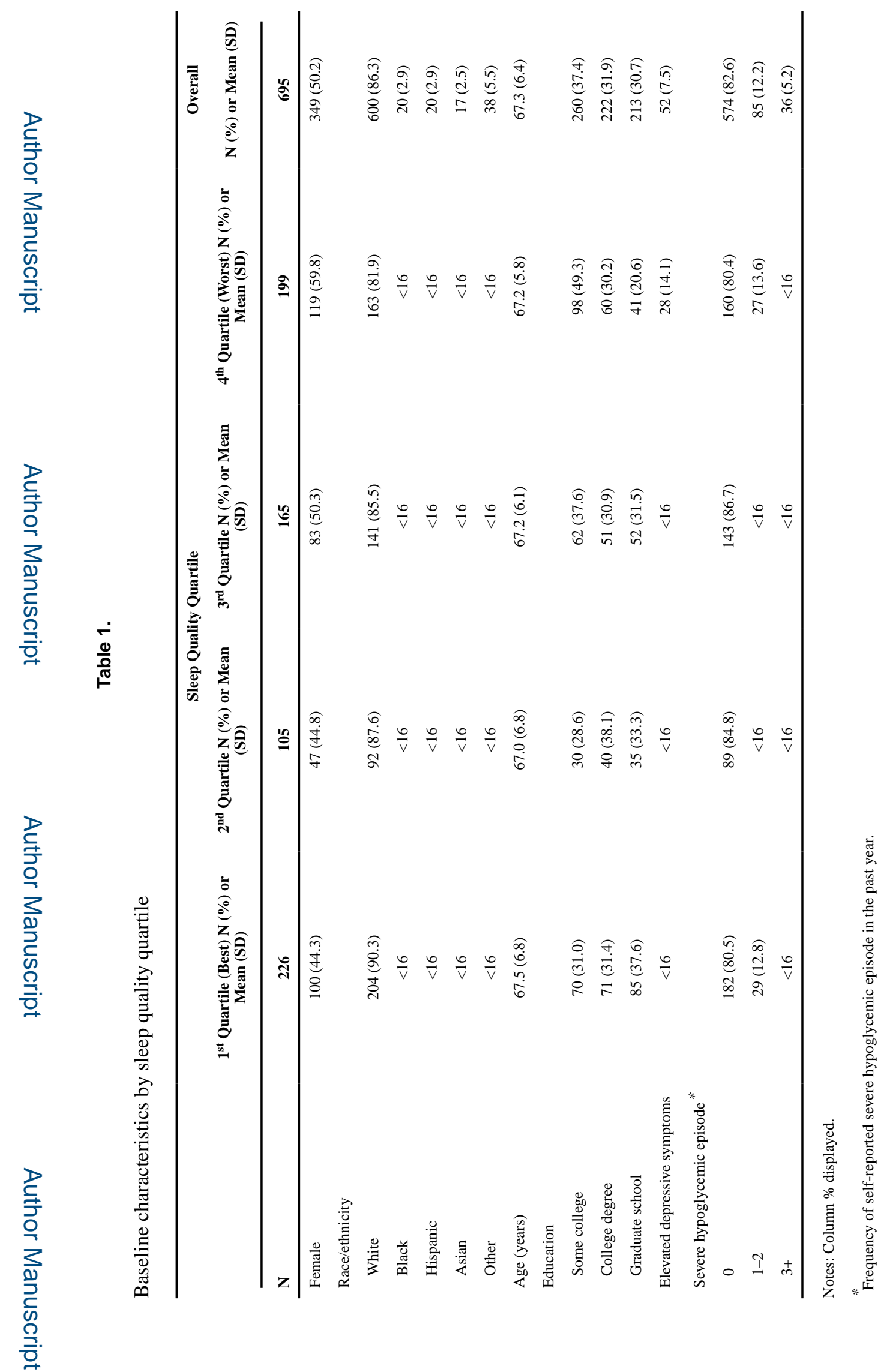

Alzheimer Dis Assoc Disord. Author manuscript; available in PMC 2021 January 01. 


\section{롤 \\ }

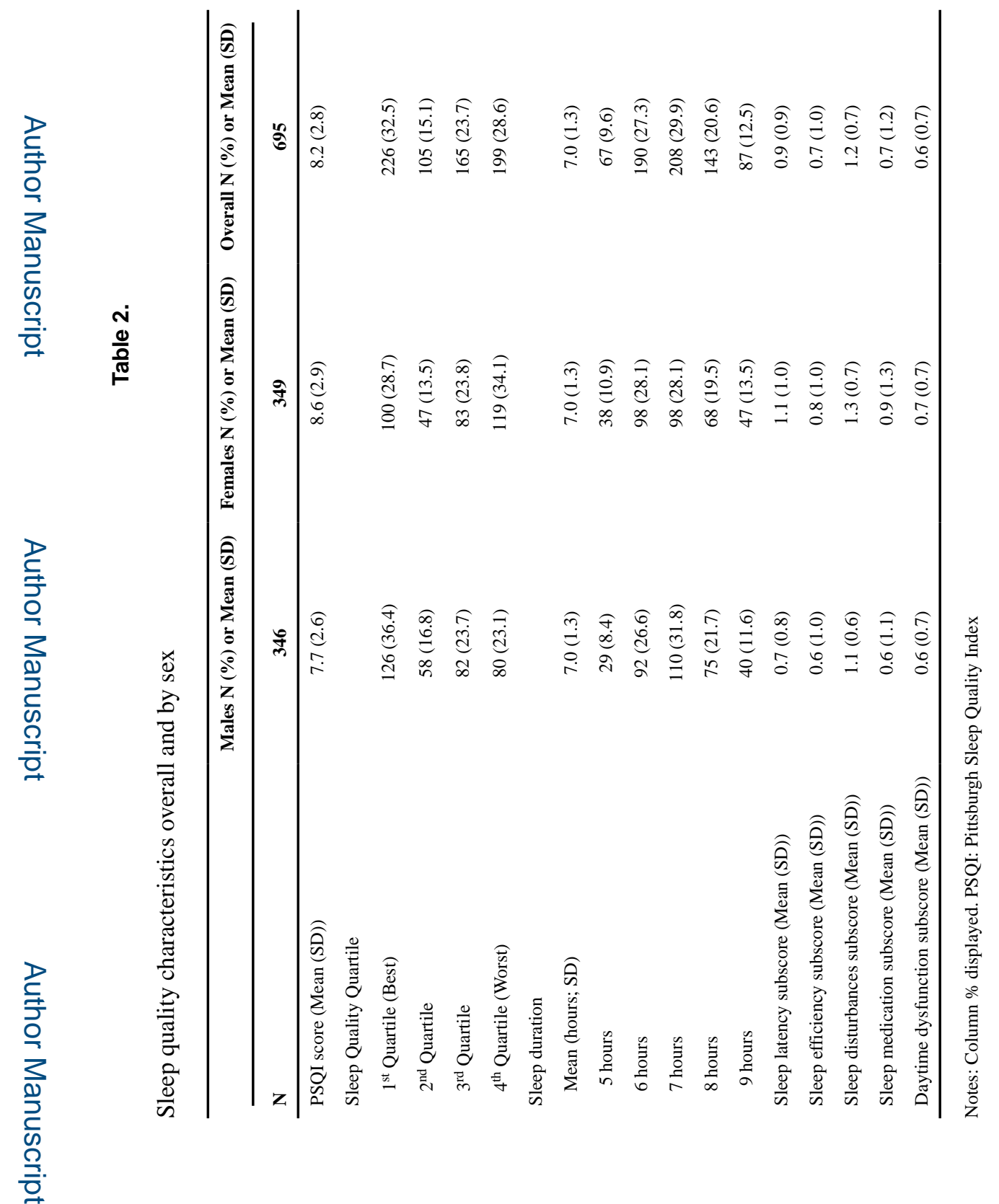

Alzheimer Dis Assoc Disord. Author manuscript; available in PMC 2021 January 01. 


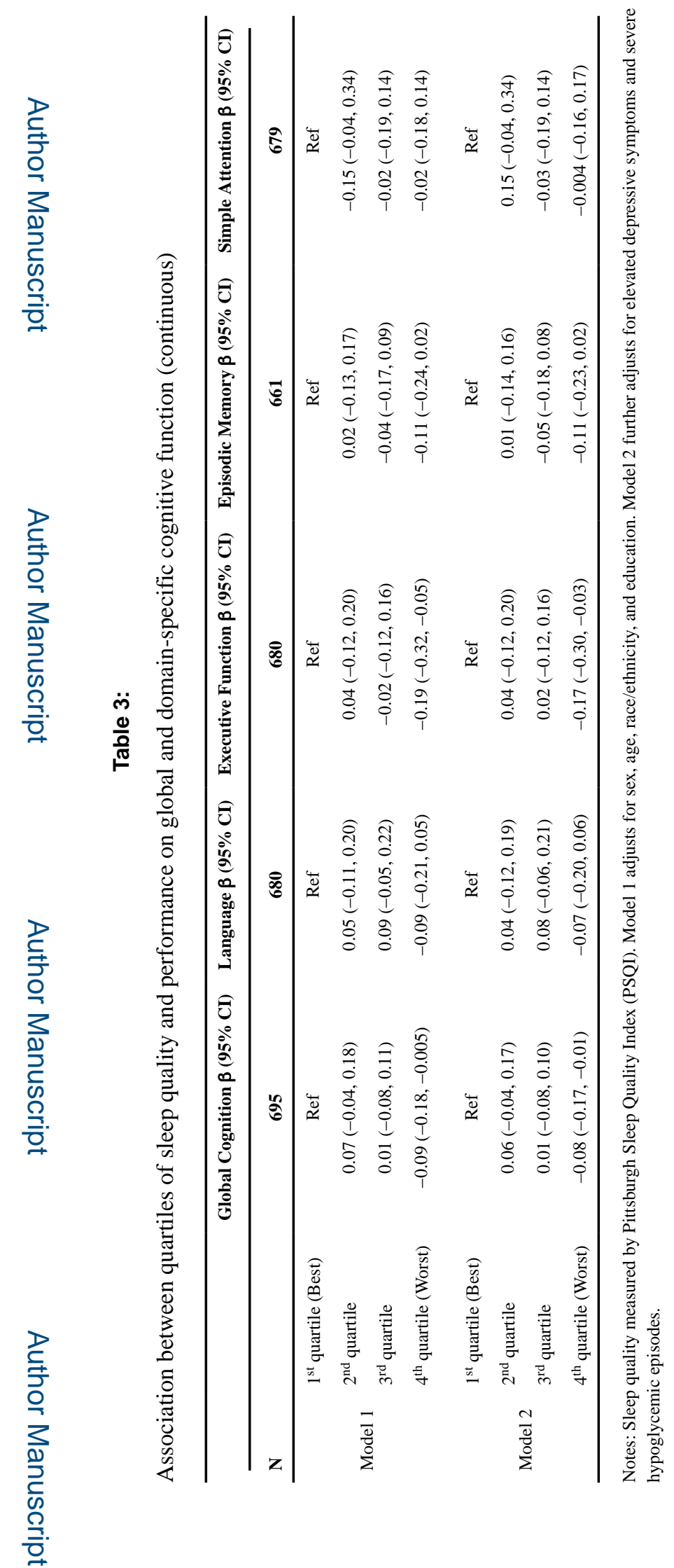

Alzheimer Dis Assoc Disord. Author manuscript; available in PMC 2021 January 01. 


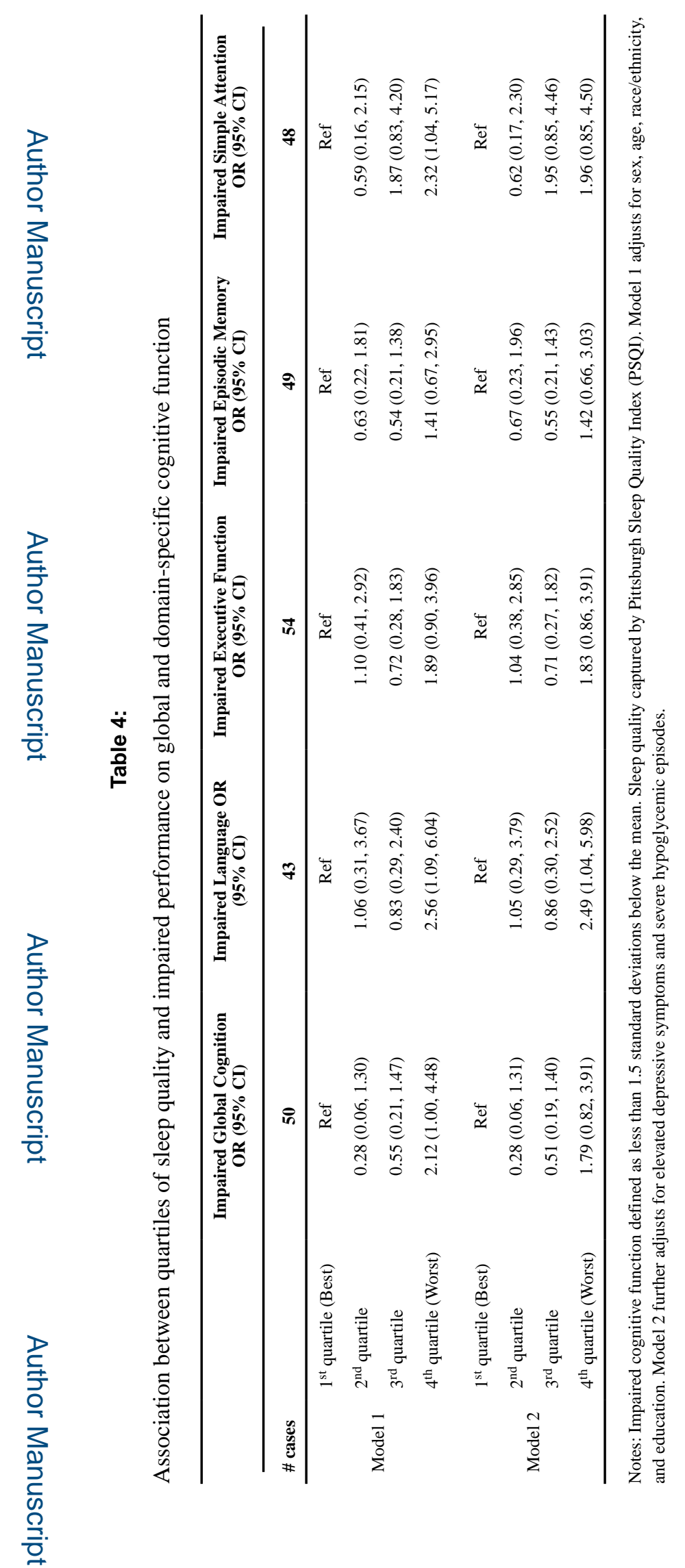

Alzheimer Dis Assoc Disord. Author manuscript; available in PMC 2021 January 01. 
Table 5:

Associations between aspects of sleep quality and performance on global cognitive function (continuous) and impaired global cognition (binary)

\begin{tabular}{|c|c|c|}
\hline & $\begin{array}{c}\text { Global Cognition } \\
\beta(95 \% \mathrm{CI})\end{array}$ & $\begin{array}{c}\text { Impaired Global Cognition } \\
\text { OR }(95 \% \mathrm{CI})\end{array}$ \\
\hline Subjective sleep quality & $0.02(-0.02,0.07)$ & $1.03(0.64,1.66)$ \\
\hline Sleep latency & $-0.01(-0.05,0.03)$ & $1.49(0.94,1.85)$ \\
\hline Sleep efficiency & $-0.01(-0.04,0.03)$ & $1.04(0.76,1.42)$ \\
\hline \multicolumn{3}{|l|}{ Sleep duration } \\
\hline Continuous (hrs) & $-0.01(-0.04,0.01)$ & $1.12(0.88,1.42)$ \\
\hline 5 hours & $0.06(-0.04,0.17)$ & $0.56(0.20,1.60)$ \\
\hline 56 hours & $0.0002(-0.7,0.07)$ & $0.83(0.41,1.67)$ \\
\hline$\leq 7$ hours & $0.06(-0.01,0.13)$ & $0.65(0.34,1.27)$ \\
\hline$\geqslant 9$ hours & $0.04(-0.07,0.15)$ & $0.84(0.27,2.62)$ \\
\hline Sleep disturbances & $-0.03(-0.08,0.02)$ & $1.30(0.82,2.06)$ \\
\hline Sleep medication use & $-0.03(-0.06,-0.002)$ & $1.24(0.97,1.58)$ \\
\hline Daytime dysfunction & $-0.07(-0.12,-0.02)$ & $1.34(0.88,2.03)$ \\
\hline
\end{tabular}

Notes: Adjusted for sex, age, race/ethnicity, education, elevated depressive symptoms, and severe hypoglycemic episodes. Impaired global cognition defined as 1.5 standard deviations below the mean. 